\title{
The impact of the COVID-19 pandemic on patients with primary Sjögren syndrome
}

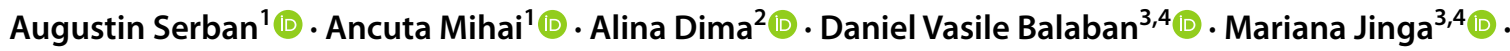 \\ Ciprian Jurcut ${ }^{1} \mathbb{D}$
}

Received: 7 July 2021 / Accepted: 5 August 2021 / Published online: 28 August 2021

(c) The Author(s), under exclusive licence to Springer-Verlag GmbH Germany, part of Springer Nature 2021

\begin{abstract}
The aim of this study was to investigate the perspective of Romanian patients with Sjögren syndrome (SS) on various aspects of the disease during the SARS-CoV-2 outbreak, including both the impact of COVID-19 on the disease itself as well as the effects of vaccination against SARS-CoV-2 in this group of patients. The study is an online questionnaire-based survey. We received responses from 137 SS patients. Regarding the general emotional status, 33 patients (24.0\%) and 47 patients (34.3\%) declared to have been sadder/depressive and more agitated/anxious during the SARS-CoV2 outbreak, respectively. During the lockdown, $49(33.7 \%)$ patients strictly and 77 patients $(56.2 \%)$ did their best to respect the home isolation measures. The income was unchanged for most of the patients (94 patients, 68.6\%). Regarding access to healthcare providers, 27 patients (18.7\%) postponed the consultation for fear of getting SARS-CoV2. In our study group, 31 patients (22.6\%) responded that they have had COVID-19. Only one patient was completely asymptomatic, while the most frequently declared symptom was weakness (84.0\%). In 17 patients among the respondents (68\%) the symptoms lasted for at least 2 weeks; the most frequent long-lasting symptoms were fatigue (40.0\%) and weakness (36.0\%). Out of all the respondents, 53 patients (41.4\%) were vaccinated against SARS-CoV2 with at least one dose. After the first dose, the most prevalent side effect was pain at the site of injection (89.2\%), followed by weakness (25.0\%) and myalgias (21.4\%). This information will be useful for developing special programs dedicated to SARS-CoV2 infection and vaccination in patients with SS and other autoimmune diseases.
\end{abstract}

Keywords SARS-CoV2 $\cdot$ COVID-19 $\cdot$ Sjögren syndrome $\cdot$ Vaccination $\cdot$ Pandemic

Augustin Serban and Ancuta Mihai have equally contributed to this work.

Alina Dima

alina_dima@outlook.com

1 Department of Internal Medicine, Dr. Carol Davila Central University Emergency Military Hospital, Bucharest, Romania

2 Department of Rheumatology, Colentina Clinical Hospital, Bucharest, Romania

3 Internal Medicine and Gastroenterology Department, Carol Davila University of Medicine and Pharmacy, Bucharest, Romania

4 Gastroenterology Department, Dr. Carol Davila Central University Emergency Military Hospital, Bucharest, Romania

\section{Background}

Sjögren syndrome (SS) is an autoimmune disease affecting primarily the lacrimal and salivary glands, leading to ocular and oral dryness. Beyond this, a large spectrum of systemic manifestations and associated comorbidities were described, including fatigue, depression, or anxiety.

Considering the increasing number of cases and the worldwide distribution, in March 2020, the World Health Organization (WHO) recognized the infection with the new severe acute respiratory syndrome (SARS) coronavirus (SARS-CoV-2) as being pandemic. Thus, in most countries with a significant number of cases, the various general (wearing masks, social distancing) or more restrictive lockdown measures adapted to each country or region were applied to stop the infection from spreading. However, beyond limiting the SARS-CoV-2 infection, these measures had a significant impact on the general quality of life, the access to some specific medications (i.e., hydroxychloroquine) and on healthcare providers of patients 
with chronic diseases, including autoimmune pathologies like SS $[1,2]$.

The aim of this study was to investigate the Romanian SS patients' perspective on the general aspects of the disease and on overall life during the SARS-CoV-2 outbreak, the impact of COVID-19 on disease symptoms and the impact of vaccination against SARS-CoV-2 in this group of patients. To the best of our knowledge, this is the first research reporting the SARS-CoV-2 pandemic's impact on SS patients in Romania.

\section{Methods}

This study is an online questionnaire-based survey and the methodology followed the available recommendations [3, 4]. The questions were related to the main aspects concerning the SARS-CoV2 outbreak in patients with SS, including the impact of COVID-19 disease and vaccination against the new coronavirus on these patients. The questions were uploaded to a dedicated online platform (https://www.surve ymonkey.com/) and distributed either via direct messaging to patients from our center's cohort or by means of our local SS patient's groups via social media platforms by one of the patient's representative. The survey was developed by the authors which are experts in the field, the usability and technical functionality were tested before the questionnaire's release to the group of patients.

The first page of the questionnaire's link explained the purpose of the research. All the included patients voluntarily decided to take part in the survey and completed it by accessing the survey link. Any participant could complete the questionnaire only once, based on the IP address. The survey was available for 1 month, between the 15th of March and the 15th of April 2021. The participation in this study was completely voluntary. Filling out the questionnaire was done after reading the first survey page and implied giving informed consent to participate in this study. The Ethics Committee of the Carol Davila University of Medicine and Pharmacy, Bucharest approved this research (No 5752/2021). No personal identification data from the questionnaire's respondents were stored.

The statistical approach was descriptive, and categorical variables were expressed as numbers and percentages. The SPSS V25 Software (IBM Corp, Armonk, NY, USA) was used.

\section{Results}

\section{General data}

The number of respondents to the online survey was 137 patients. The general characteristics of our study group are shown in the Supplementary Table. The mean age at inclusion was $47.9 \pm 10.9$ years and the mean disease duration was $5.9 \pm 4.8$ years. We noted a large predominance of the female sex (94.9\%). Regarding the ongoing treatment, 119 patients $(86.8 \%)$ were treated with artificial tears, 30 patients (21.9\%) were on mouth topical drugs, and 94 patients $(68.6 \%)$ on hydroxychloroquine.

\section{Impact of the SARS-CoV2 outbreak in patients with SS}

The reported impact of the SARS-CoV2 outbreak is presented in Table 1. Regarding general emotional status, 38 patients $(27.7 \%)$ did not observe any difference, while 33 patients $(24.0 \%)$ and 47 patients $(34.3 \%)$ were sadder/ depressive and more agitated /anxious, respectively. A very limited number of patients (2 patients; $1.4 \%$ ) sought the help of a psychologist or psychiatrist. Also, only a minority of patients declared to have been more relaxed or calmer ( 8 patients; 5.8\%), while 13 patients (9.4\%) were more rested during the pandemic outbreak.

Moreover, during the lockdown, 49 (35.7\%) patients strictly and 77 patients (56.2\%) did their best to respect the home isolation measures, leaving their home only for good reasons.

The income was unchanged for most of the patients (94 patients; $68.6 \%$ ); however, as many as $21.1 \%$ (29 patients) earned less than before the outbreak. No changes in professional activity were noted in 51 patients (37.2\%), while other patients were working for the same employer but from home (22 patients; $16.0 \%$ ) or with more days off and reduced salary (9 patients; 6.5\%). Two patients (1.4\%) lost their job and 11 patients $(8.0 \%)$ resigned.

Regarding the access to healthcare providers, 27 patients (18.7\%) postponed the consultation for fear of getting SARSCoV2 and 12 patients (8.7\%) needed healthcare, but could not access the public hospital services.

\section{Impact of COVID-19 in patients with SS}

In our study group, 31 patients $(22.6 \%)$ responded that they have had COVID-19, out of which 25 patients responded to the questions related to the COVID-19 impact (rate of response $80.6 \%$ ). Among the respondents, seven patients $(28 \%)$ thought that they got the infection at work, while five respondents cannot pinpoint the source of the infection. The COVID-19 symptoms present during the infection are listed in Table 2. Only one patient (4.0\%) was completely asymptomatic, while the most frequently declared symptom was weakness (21 patients; $84.0 \%)$. The duration of the symptoms was less than one week in only one patient $(4.0 \%)$, while in 17 patients $(68.0 \%)$ the symptoms lasted for at least 2 weeks. The 
Table. 1 Impact of the SARS$\mathrm{CoV} 2$ outbreak on general and socio-economic parameters in patients with Sjogren's syndrome

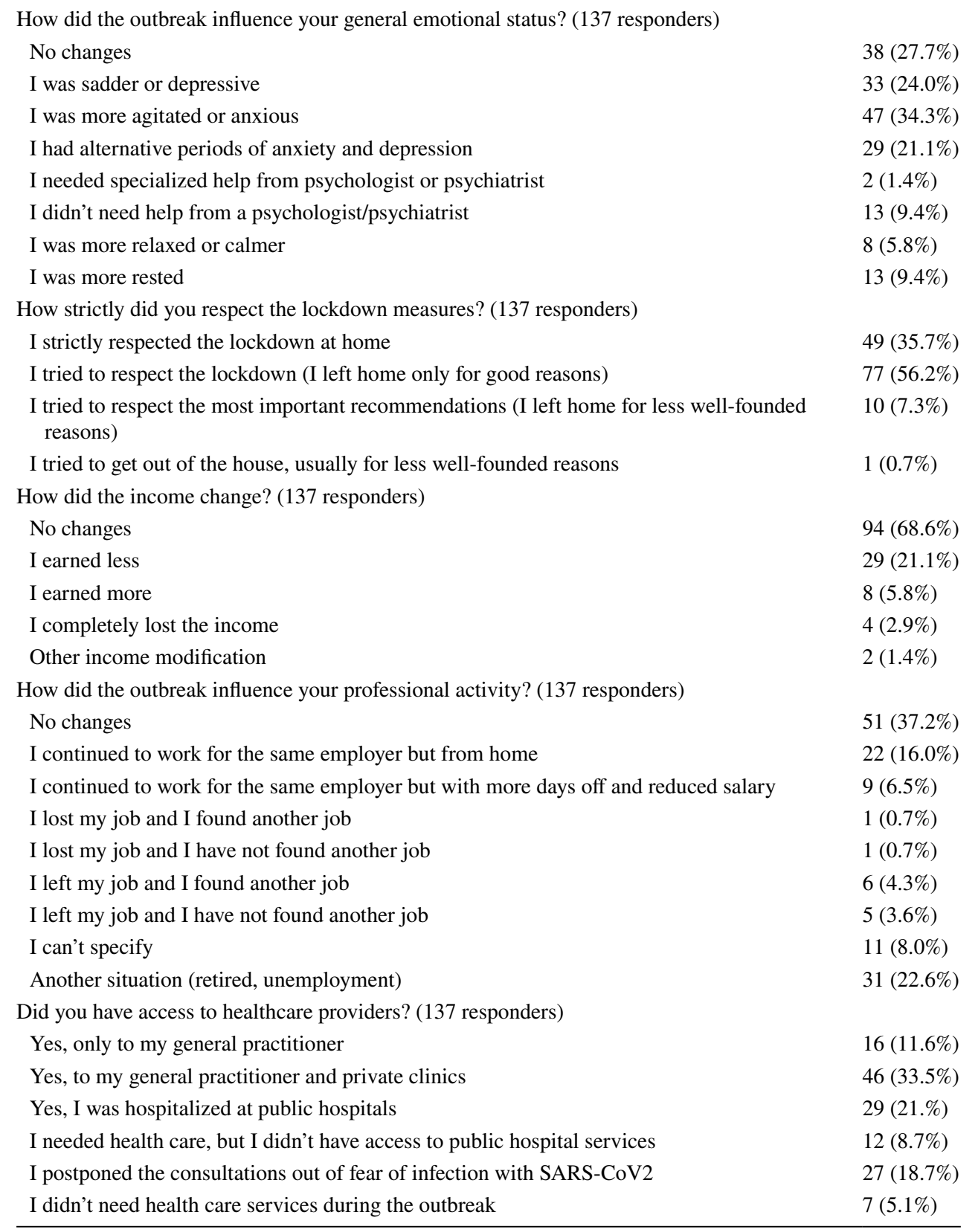

most frequent long-lasting symptoms were weakness (9 patients; $36.0 \%)$ and fatigue (10 patients; $40.0 \%)$. Three patients $(12.0 \%)$ were hospitalized, none of them requiring admission to ICU or intubation. Regarding the specific SS symptoms, 12 patients $(48.0 \%)$ reported no aggravation, while 9 patients $(36.0 \%), 6$ patients $(24.0 \%)$, and 8 patients $(32.0 \%)$ reported more severe eye dryness, more severe oral dryness and oral aphthae/ulcerations, respectively. The most persistent symptoms, lasting for weeks or months after the infection, were more severe oral dryness (10 patients, $40.0 \%$ ) and nasal dryness $(32.0 \%)$. After the COVID-19 acute infection, 7 patients $(28.0 \%)$ declared more frequent use of artificial tears.

\section{Anti-SARS-Cov2 vaccine in patients with SS}

Among the respondents, 53 patients (41.4\%) were vaccinated against SARS-CoV2 with at least one dose, but only 28 patients (rate of response $52.8 \%$ ) responded to the questions regarding vaccination`s impact. After the first dose, the most prevalent side effect was pain at the site of injection (25 patients; $89.2 \%$ ), followed by weakness (7 patients; $25.0 \%$ ) and myalgias (6 patients; $21.4 \%$ ) (Table 3). Only three vaccinated patients $(10.7 \%)$ reported that their dryness-related symptoms were aggravated after the vaccine. 
Table.2 Impact of the COVID disease in patients with Sjogren's syndrome
Did you have COVID-19? (137 responders)

Yes

$31(22.6 \%)$

No

$106(77.3 \%)$

Where do you think you got it from? (25 responders)

From my job

$7(28.0 \%)$

From a family member living together

$5(20.0 \%)$

From my entourage, not living together

$4(16.0 \%)$

From my flat/room mate

$2(8.0 \%)$

From transport means (bus/tram/metro/taxi)

$3(12.0 \%)$

I do not know

$5(20.0 \%)$

School/college/university, hospital/general practitioner, shopping, elevators

$0(0.0 \%)$

Symptoms during infection (25 responders)

No symptoms

$1(4.0 \%)$

Feeling weak

$21(84.0 \%)$

Myalgias

$16(64.0 \%)$

Anosmia

$15(60.0 \%)$

Headache

$14(56.0 \%)$

Severe fatigue

$12(48.0 \%)$

Cough

$12(48.0 \%)$

Ageusia

$11(44.0 \%)$

Fever

$11(44.0 \%)$

Resting dyspnea

$8(32.0 \%)$

Effort dyspnea

$7(28.0 \%)$

Diarrhea

$7(28.0 \%)$

Nausea and vomiting

$4(16.0 \%)$

Rhinorrhea

$1(16.0 \%)$

Sore throat

$2(8.0 \%)$

Duration of symptoms (25 responders)

No symptoms

$1(4.0 \%)$

$1(4.0 \%)$

$6(24.0 \%)$

$9(36.0 \%)$

$5(20.0 \%)$

$3(12.0 \%)$

$>4$ weeks

Symptoms persisting more than 2 weeks ( 25 responders)

No symptoms $>2$ weeks

$5(20.0 \%)$

Feeling weak

$9(36.0 \%)$

Myalgias

$7(28.0 \%)$

Anosmia

$5(20.0 \%)$

Headache

$3(12.0 \%)$

Severe fatigue

$10(40.0 \%)$

Cough

$1(4.0 \%)$

Ageusia

$4(16.0 \%)$

Resting dyspnea

$3(12.0 \%)$

Effort dyspnea

$5(20.0 \%)$

$1(4.0 \%)$

$1(4.0 \%)$

Rhinorrhea

$20(80.0 \%)$

$2(8.0 \%)$

$2(8.0 \%)$

Yes, I was hospitalized with severe symptoms

$1(4.0 \%)$ 
Table.2 (continued)

$\begin{array}{ll}\text { Symptoms of SS during infection (25 responders) } & \\ \text { More severe eye dryness } & 9(36.0 \%) \\ \text { More severe oral dryness } & 6(24.0 \%) \\ \text { New or more severe nasal dryness } & 9(36.0 \%) \\ \text { New or more severe genital dryness } & 2(8.0 \%) \\ \text { Oral aphthae or ulcerations } & 8(32.0 \%) \\ \text { Corneal ulcerations } & 1(4.0 \%) \\ \text { No aggravation of symptoms } & 12(48 \%) \\ \text { Symptoms of SS persisting for weeks-months after infection (25 responders) } & \\ \text { More severe eye dryness } & 7(28.0 \%) \\ \text { More severe oral dryness } & 10(40.0 \%) \\ \text { New or more severe nasal dryness } & 8(32.0 \%) \\ \text { No aggravation of symptoms } & 11(44.0 \%) \\ \text { After the infection you used the artificial tears (25 responders) } & \\ \text { More frequently } & 7(28.0 \%) \\ \text { Less frequently } & 4(16.0 \%) \\ \text { No modification } & 14(56.0 \%)\end{array}$

Table.3 Anti-SARS-CoV2 vaccination in patients with Sjogren's syndrome
Were you vaccinated against SARS-CoV2? (128 responders)

Yes

No

Did you have side effects after the $1^{\text {st }}$ dose? (28 responders)

No side effects

$3(10.7 \%)$

Pain at the injection site

Redness at the injection site

$1(3.5 \%)$

Cervical or axillar adenopathies

$2(7.1 \%)$

Weakness

$7(25.0 \%)$

Headache

$4(14.2 \%)$

Myalgias

$6(21.4 \%)$

Joint pain

$3(10.7 \%)$

Fever

$3(10.7 \%)$

Chills

$2(7.1 \%)$

Dizziness

$4(14.2 \%)$

If you were not vaccinated, this is because ( 74 responders)

I was not assured that I can receive the vaccine in the context of SS $30(40.5 \%)$

I did not have an appointment, but I intend to get vaccinated $\quad 19(25.6 \%)$

I did not understand how the appointment works $1(1.3 \%)$

I consider that the side effects of the vaccine are not fully known $29(39.1 \%)$

$\begin{array}{ll}\text { I think that vaccines do not work } & 2(2.7 \%)\end{array}$

I had been infected with COVID-19 and I think that the vaccine is unnecessary in this $\quad 7(9.4 \%)$ context

Were the eye or oral dryness symptoms modified after the vaccine? (28 responders)

No, I did not notice any changes

Yes, the dryness symptoms were aggravated

Yes, the dryness symptoms were improved 
The main reason for not getting vaccinated was the fact that the patients were insecure about receiving the SARS$\mathrm{CoV} 2$ vaccine while having a diagnosis of SS (30 patients; $40.5 \%)$.

\section{Discussion}

This survey presented the self-perception of patients with SS regarding the SARS-CoV2 outbreak and included 137 respondents. The general characteristics of the study group (i.e., gender distribution, mean age of the patients) are compatible with similar SS cohorts.

There are few studies evaluating the emotional impact of the SARS-CoV2 outbreak in patients with SS. The general emotional status was unchanged in $27.7 \%$ of respondents. However, many patients were sadder or depressive (24.0\%) and agitated or anxious (34.3\%) during the outbreak.

In the study of Giardina et al., evaluating the impact of the outbreak on patients with SS, 32.6\% never left home and $65.3 \%$ left home for necessities [2]. As in this study, similar adherence to lockdown measures were also reported in our survey, namely $35.7 \%$ respected strictly the lockdown measures, while $56.2 \%$ left home with caution, only for important reasons.

Regarding the professional and financial aspects during the SARS-CoV2 outbreak, there is a lack of data in patients with SS. Most of the patients reported a similar income, $21.1 \%$ of patients reported a lower income, $8.0 \%$ of patients resigned and $1.4 \%$ of patients lost their job. Income decrease frequency is similar to that reported in the general Romanian population by Radulescu et al. [5], who also found that the COVID-19 pandemic reduced the number of employees on the national market.

The lockdown measures negatively impacted some specific aspects related to access to outpatient clinic consultations, hospitalizations or drug availability [6]. In our study, $18.7 \%$ of patients postponed the consultation out of fear of infection, but generally the patients had access to their general practitioner, private clinics, and public hospitals. In contrast, in the study of Carubbi et al. [7], 95.0\% of patients with SS had their rheumatology consultation canceled and $83.0 \%$ of patients had their SS related tests canceled. These discrepant results might be in part due to the fact that in the first phase of the outbreak, when the study of Carubbi et al. was performed, the lockdown measures were probably more restrictive. However, our survey was conducted 1 year after the beginning of the outbreak.

In our study, $22.6 \%$ of patients with SS reported that they have had COVID-19. Previously, the COVID-19 prevalence in patients with SS was reported to be less than $1.0 \%$ [8]. In our study, the self-reported prevalence is higher than that reported in Romanian general population, but we have to mention that there are presumptions of an important number of subjects from the general population infected but not tested for SARS-CoV2 and, therefore, not confirmed [9]. One Italian study showed a higher frequency of COVID-19 occurrence in patients with autoimmune systemic diseases when compared to the general population, especially in patients without ongoing immunosuppressive treatments [10].

Regarding the symptoms, only $4.0 \%$ of the respondents were completely asymptomatic. The most important symptom during the infection was weakness $(84.0 \%)$. More than one third of the patients questioned reported general symptoms like weakness and fatigue as long-lasting post-COVID-19 issues.

During the course of the disease, there were patients reporting the aggravation of dryness symptoms $(36.0 \%$ for eye dryness and $24.0 \%$ for oral dryness), in some cases persisting weeks or months after the infection. This led to more frequent use of artificial tears after the infection.

In our cohort, $41.4 \%$ of patients were vaccinated with at least one dose of vaccine, above the reported vaccination rate in the general population of Romania at the end of the survey $\left(15^{\text {th }}\right.$ of April 2021) [11].

Regarding reported side effects after one dose, the most prevalent side effects were only mild ones, like pain at the site of injection, weakness and myalgias, comparable with that reported in the general population [12].

Only three of the vaccinated patients $(10.7 \%)$ reported aggravation of dryness symptoms after the vaccine. One of the most important concerns related to the SARS-CoV2 vaccine in patients with autoimmune disease was the fear of disease flare after the injection. In an online survey-based study, Felten et al. reported three clusters of opinions regarding vaccination in patients with autoimmune diseases and the fear of disease flare was cited among other patients concerns $(36.6 \%$ vs $80.8 \%$ in patients willing vs unwilling to be vaccinated, $\mathrm{p}<0.0001)$ [13].

Understanding the reasons for not getting vaccinated, the perception and knowledge of patients are important aspects during the vaccination campaign [14]. Our survey showed a lack of information regarding the safety and efficiency of the anti-SARS-CoV2 vaccine, most of the vaccination refusals being due to fear of disease-related contraindications to vaccination in SS patients as well as to the lack of trust in the vaccine's utility. The shortage of information regarding the interaction of SARS-CoV2 vaccine with autoimmune diseases [6], especially the fear of background disease aggravation and lack of correct immune response and protection [13], determined medical organizations to promote educational materials [6]. It should be noted that similar fears were reported by physicians as well $[12,15]$. 


\section{Limits of the study}

The current study has some limitations. The strictly online distribution of the survey is one of them. However, the distribution was done by a SS patients association representative in their on-line group on social media, as well as by direct messaging to the members of our center's cohort. This methodology enlarged the respondent base. Another limitation might be the small number of SS patients participating in the survey. However, studies with similar research themes and designs did not include a higher number of patients [7, 8]. Also, a lower response rate to on-line forms when compared to face to face completed questionnaires is acknowledged [16]. Even if we tried to limit the respondent bias by anonymously and self-administrated questionnaire and use of forced-choice items and neutral questions, we cannot completely evaluate the rate of response bias on vulnerable themes like the pandemic influence over the emotional status or lockdown measures. The questionnaire was distributed via communication methods available only to patients from our cohort and the local association of SS patients, however, being an open link, we cannot completely rule out answers from outside the group. Also, this research does not offer a comparison with the general population.

\section{Conclusions}

To conclude, we present the results of an on-line survey reflecting the perception of Romanian patients with SS on the SARS-CoV2 outbreak, COVID-19 infection and vaccination. SS does not appear to be a risk factor for severe COVID-19, moreover, the vaccination against COVID-19 in our group of patients appeared to be safe. This information will be useful for developing special programs dedicated to SARS-CoV2 infection and vaccination in patients with SS and other autoimmune diseases.

Supplementary Information The online version contains supplementary material available at https://doi.org/10.1007/s00296-021-04967-4.

Acknowledgements We thank Nadia Radulescu, representative of the patients' association, for her support in survey distribution.

Author contributions All authors had substantial contribution to the acquisition and analysis of the data presented in this article. All authors revised and agreed on the final version of the work to be published. All authors take full responsibility in regard to all aspects of this work. Any questions that may be raised in the future concerning the integrity or this article will be evaluated and properly addressed by the authors.

Funding No specific grant from any public agency, commercial or notfor-profit sectors was received for the presented research.

\section{Declarations}

Conflict of interest None of the authors has any conflict of interest that would constitute a conflict of interest with the current study.

Disclaimer stating No part of the research including tables and supplementary files, are copied, or published elsewhere.

\section{References}

1. Sloan M, Gordon C, Harwood R et al (2020) The impact of the COVID-19 pandemic on the medical care and health-care behaviour of patients with lupus and other systemic autoimmune diseases: a mixed methods longitudinal study. Rheumatol Adv Pract. https://doi.org/10.1093/rap/rkaa072

2. Giardina F, Izzo R, Gattamelata A et al (2021) COVID-19 in Italian Sjögren's syndrome patients: a monocentric study. Rheumatol Int 41:235-236. https://doi.org/10.1007/s00296-020-04722-1

3. Eysenbach $\mathrm{G}$ (2004) Improving the quality of web surveys: the checklist for reporting results of Internet E-Surveys (CHERRIES). J Med Internet Res. https://doi.org/10.2196/jmir.6.3.e34

4. Gaur PS, Zimba O, Agarwal V et al (2020) Reporting survey based studies-a primer for authors. J Korean Med Sci 35:1-15. https://doi.org/10.3346/jkms.2020.35.e398

5. Radulescu CV, Ladaru G-R, Burlacu S et al (2021) Impact of the COVID-19 Pandemic on the Romanian Labor Market. Sustain 13:271. https://doi.org/10.3390/su13010271

6. Talarico R, Aguilera S, Alexander T et al (2021) The impact of COVID-19 on rare and complex connective tissue diseases: the experience of ERN ReCONNET. Nat Rev Rheumatol 17:177-184. https://doi.org/10.1038/s41584-020-00565-Z

7. Carubbi F, Alunno A, Ferri C et al (2020) The impact of SARSCoV-2 outbreak on primary sjögren's syndrome: an italian experience. Front Med 7:1-5. https://doi.org/10.3389/fmed.2020.608728

8. Brito-Zerón P, Melchor S, Seror R et al (2021) SARS-CoV-2 infection in patients with primary Sjögren syndrome: characterization and outcomes of 51 patients. Rheumatology (Oxford) 60:2946-2957. https://doi.org/10.1093/rheumatology/keaa748

9. COVID Live Update: 194,784,770 Cases and 4,174,363 Deaths from the Coronavirus-Worldometer. https://www.worldometers. info/coronavirus/. Accessed 26 Jul 2021.

10. Ferri C, Giuggioli D, Raimondo V et al (2020) COVID-19 and rheumatic autoimmune systemic diseases: report of a large Italian patients series. Clin Rheumatol 39:3195-3204. https://doi.org/10. 1007/s10067-020-05334-7

11. Situația vaccinării în România | Vaccinare Covid. https://vacci nare-covid.gov.ro/situatia-vaccinarii-in-romania/. Accessed $26 \mathrm{Jul}$ 2021.

12. Dima A, Balaban DV, Jurcut C et al (2021) Perceptions of Romanian physicians on lockdowns for Covid-19 prevention. Healthcare 9:95. https://doi.org/10.3390/healthcare9010095

13. Felten R, Dubois M, Ugarte-Gil MF et al (2021) Vaccination against COVID-19: expectations and concerns of patients with autoimmune and rheumatic diseases. Lancet Rheumatol 3:e243e245. https://doi.org/10.1016/S2665-9913(21)00039-4

14. Leão T, Amorim M, Fraga $S$ et al (2021) What doubts, concerns and fears about COVID-19 emerged during the first wave of the pandemic? Patient Educ Couns 104:235-241. https://doi.org/10. 1016/j.pec.2020.11.002

15 Dima A, Balaban DV, Jurcut C et al (2020) Physicians' perspectives on COVID-19: an international survey. Healthcare 8:250. https://doi.org/10.3390/healthcare 8030250 
16. Arámburo-Gálvez JG, Carvalho Gomes IC, André TG et al (2019) Translation, cultural adaptation, and evaluation of a Brazilian Portuguese questionnaire to estimate the self-reported prevalence of gluten-related disorders and adherence to gluten-free diet. Medicina (Kaunas) 55:593. https://doi.org/10.3390/medicina55 090593
Publisher's Note Springer Nature remains neutral with regard to jurisdictional claims in published maps and institutional affiliations. 